\title{
OGLE-TR-211 - a new transiting inflated hot Jupiter from the OGLE survey and ESO LP666 spectroscopic follow-up program ${ }^{\star}$
}

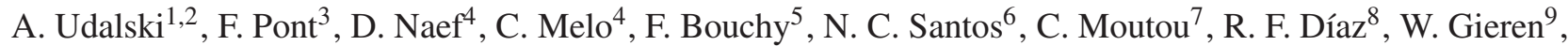 \\ M. Gillon ${ }^{3}$, S. Hoyer ${ }^{10}$, M. Mayor ${ }^{3}$, T. Mazeh ${ }^{11}$, D. Minniti ${ }^{12}$, G. Pietrzyński ${ }^{1,2,9}$, D. Queloz ${ }^{3}$, S. Ramirez ${ }^{5}$, \\ M. T. Ruiz ${ }^{10}$, A. Shporer ${ }^{11}$, O. Tamuz ${ }^{11}$, S. Udry ${ }^{3}$, M. Zoccali ${ }^{12}$, M. Kubiak ${ }^{1,2}$, M. K. Szymański ${ }^{1,2}$, I. Soszyński $^{1,2}$, \\ O. Szewczyk ${ }^{1,2,9}$, K. Ulaczyk ${ }^{1,2}$, and Ł. Wyrzykowski ${ }^{2,13}$
}

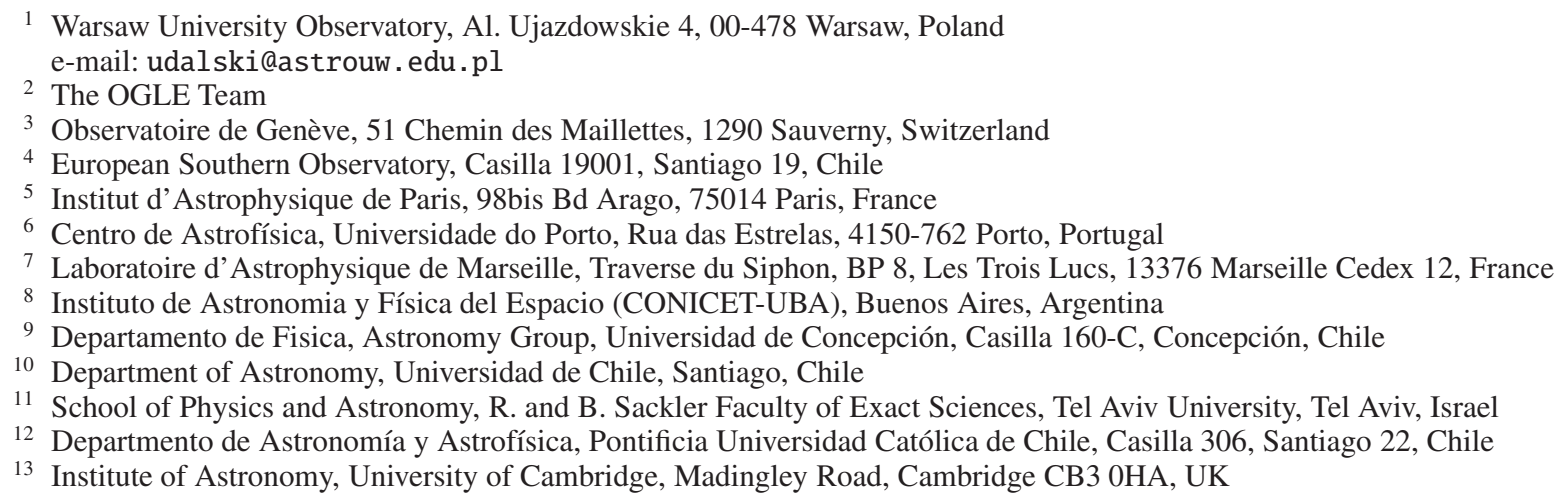

Received 26 November 2007 / Accepted 4 February 2008

\section{ABSTRACT}

\begin{abstract}
We present results of the photometric campaign for planetary and low-luminosity object transits conducted by the OGLE survey in the 2005 season (Campaign \#5). About twenty of the most promising candidates discovered in these data were subsequently verified spectroscopically with the VLT/FLAMES spectrograph.

One of the candidates, OGLE-TR-211, reveals clear changes of radial velocity with a small amplitude of $82 \mathrm{~m} / \mathrm{s}$, varying in phase with photometric transit ephemeris. Further analysis confirms the planetary nature of this system. Follow-up precise photometry of OGLE-TR-211 with VLT/FORS, together with radial velocity spectroscopy, supplemented with high-resolution, high S/N VLT/UVES spectra allowed us to derive parameters of the planet and host star. OGLE-TR-211b is a hot Jupiter orbiting an F7-8 spectral type dwarf star with a period of 3.68 days. The mass of the planet is equal to $1.03 \pm 0.20 M_{\text {Jup }}$, while its radius $1.36_{-0.09}^{+0.18} R_{\text {Jup }}$. The radius is about 20\% larger than the typical radius of hot Jupiters of similar mass. OGLE-TR-211b is, then, another example of inflated hot Jupiters - a small group of seven exoplanets with large radii and unusually low densities - objects that are a challenge to the current models of exoplanets.
\end{abstract}

Key words. planetary systems - stars: individual: OGLE-TR-211

\section{Introduction}

The discovery of the first transit of an extrasolar planet around HD 209458 (Charbonneau et al. 2000; Henry et al. 2000) opened a new era in the extrasolar planet field - the epoch of searches for extrasolar planets with large-scale photometric surveys. While this channel of finding exoplanets developed relatively slowly in the first couple of years after that discovery, the past two years brought fast acceleration due to the search methods maturing. At present about 30 transiting planets are known (cf. http: //WWW . inscience.ch/transits/) and they constitute about $10 \%$ of all known exoplanets.

Transiting planets play an especially important role among the known exoplanets. These are the only objects for which the

* Based on observations made with the FORS1 camera and the FLAMES/UVES spectrograph at the VLT, ESO, Chile (program 07.C0706, 076.C-0122, and 177.C-0666) and 1.3-m Warsaw Telescope at Las Campanas Observatory, Chile. most important parameters (e.g., radius, mass, density) can be precisely derived from observations, thereby providing direct comparison with models and thus allowing a better understanding of the exoplanet structure and evolution. Also, a wide variety of follow-up observations can be performed on transiting system to allow, for example, studies of planetary atmospheres and search for other planetary companions, etc.

Transiting planets are discovered via two channels: photometric follow-up of spectroscopically found exoplanets and the classical transit method approach, i.e., large scale photometric surveys providing transiting candidates that have to then be verified and confirmed spectroscopically. Both approaches have been successful in providing examples of very wide diversity among transiting planets - regular hot Jupiters, very hot Jupiters on extremely short 1-2 day period orbits, inflated objects with radii much larger than expected from modeling, small Neptunesized objects, or very massive planets on eccentric orbits. Large numbers of new extrasolar transiting planets are expected to be 
discovered in the next couple of years from space missions like Corot or Kepler, in particular small-size planets undetectable in ground-based searches.

The Optical Gravitational Lensing Experiment (OGLE) was the first successful photometric survey to discover a large number of transiting candidates that were subsequently verified spectroscopically (Udalski et al. 2002a,b,c, 2003). Five of these candidates, OGLE-TR-56, OGLE-TR-113, OGLE-TR-132, OGLETR-111, and OGLE-TR-10, turned out to be extrasolar planetary systems (Konacki et al. 2003; Bouchy et al. 2004; Konacki et al. 2004; Pont et al. 2004; Bouchy et al. 2005; Konacki et al. 2005), the first extrasolar planets discovered with the transit method. Moreover, two planet-sized stars with the lowest known masses were also found (Pont et al. 2005b, 2006a). In addition, the OGLE photometric transit campaigns provided huge amounts of observational material allowing better understanding of the problems of photometric transit searches, data systematics, etc. (Gould et al. 2006; Pont et al. 2006b).

The OGLE transit campaigns have been conducted every year since 2001. Results of the spectroscopic follow-up of candidates discovered during Campaigns \#1 (2001) and \#2 (2002) were published by Bouchy et al. (2005) and Pont et al. (2005a), respectively. Recently, analysis of the near threshold candidates from Campaign \#2 led to the discovery of the sixth OGLE transiting exoplanet, OGLR-TR-182 (Pont et al. 2007).

In this paper we present results of OGLE Campaign \#5 conducted in 2005 and spectroscopic follow-up of the best transiting candidates detected in these data. A new transiting extrasolar planetary system OGLE-TR-211 was found among them. In the following sections we provide details of the photometric and spectroscopic observations of OGLE new candidates and derive parameters of a planetary companion in OGLE-TR-211 system.

\section{OGLE planetary transit campaign \#5}

OGLE planetary transit campaigns became a standard part of the OGLE observing schedule. About $75 \%$ of observing time was devoted to this sub-project of the OGLE survey every southern fall - from February to April. The OGLE planetary campaign \#5 was conducted by OGLE in the 2005 observing season from February 2, 2005 to June 23, 2005. Observations were carried out with the 1.3-m Warsaw Telescope at Las Campanas Observatory, equipped with the $8192 \times 8192$ pixel CCD mosaic camera (Udalski 2003). The observing strategy was similar to previous campaigns, in particular \#3 and \#4 (Udalski et al. 2004). Because the experience gathered during the spectroscopic observations of the first OGLE candidates indicated some trouble with reasonable spectroscopic follow-up of the faintest objects from typical OGLE candidate lists, the exposure time during campaign \#5 was shortened to $120 \mathrm{~s}$. This allowed one more field to be added therby increasing the number of observed fields to four and covering about 1.4 square degrees of the Galactic disk regions in the Carina constellation. The cadence time for each of these four fields was about $16 \mathrm{~min}$, and their equatorial coordinates are listed in Table 1. All observations were collected through the $I$-band filter. The median seeing of the all observations was about 1.1 arcsec. Altogether, about 1320 images of each of the fields were secured during the campaign. Also a few $V$-band observations were taken for color information and CMD construction.

Collected data were reduced in a similar way to those of Campaigns \#3 and \#4 (Udalski et al. 2004). The selection of transit candidates was also performed similarly: all non-variable objects, that is, those with the rms of the average magnitude of
Table 1. Equatorial coordinates of the fields observed during the OGLE Campaign \#5.

\begin{tabular}{ccc}
\hline \hline Field & RA(J2000) & Dec(J2000) \\
\hline CAR107 & $10: 47: 15$ & $-62: 00: 25$ \\
CAR108 & $10: 47: 15$ & $-61: 24: 35$ \\
CAR109 & $10: 42: 10$ & $-62: 10: 25$ \\
CAR110 & $10: 42: 15$ & $-61: 34: 35$ \\
\hline
\end{tabular}

all observations smaller than $0.015 \mathrm{mag}$, were subject to the detrending algorithm of Kruszewski \& Semeniuk (2003) and then to a transit search procedure using the BLS algorithm of Kovács et al. (2002). Altogether, light curves of about 50000 stars were analyzed. The list of transiting candidates was prepared after careful visual inspection of objects that passed the BLS criteria. Clear false cases triggered, for example, by noisy light curves were removed. We also excluded objects with any depth of transits greater than $50 \mathrm{mmag}$, with a clear signature of ellipsoidal effect indicating massive secondary or those with clear V-shape of transits indicating grazing eclipses of a binary star. The minimum number of individual transits collected during the campaign was set to three. For all the candidates, the limits on the size of a transiting companion were calculated as in Udalski et al. (2004). All candidates with a companion whose lower limit of radius was larger than $0.2 R_{\odot}$ were removed from the final list.

Table 2 lists the transit candidates from the OGLE Campaign \#5 that passed the photometric search criteria. The naming convention follows the standard OGLE convention, i.e., OGLE-TRNNN. About twenty candidates for transiting planetary systems were found. These objects were selected as targets for spectroscopic follow-up observations conducted under the LP666 program. The photometry of all selected candidates is available from the OGLE Internet archive at $\mathrm{ftp}$ : //ftp . astrouw . edu . pl/ogle/ogle3/transits/tr201-219/

\section{Spectroscopic follow-up}

Spectroscopic follow-up observations of the OGLE Campaign \#5 transit candidates were carried out during three observing slots allocated to the LP666 program in April/May 2006, February 2007, and April 2007 on VLT with the FLAMES spectrograph. Also, part of the Geneva group observing time under program 07.C-0706 on the same instrument in February 2006 was used. Unfortunately the weather conditions during all these observing runs were exceptionally unfavorable with many cloudy or large seeing nights ( $>2$ arcsec), considerably limiting the results. In particular after the initial screening of all candidates, only the most promising objects were observed further. The remaining objects, in contrast to the previous follow-up observations (Bouchy et al. 2004; Pont et al. 2005a), were left without any full characterization. The strategy of the spectroscopic follow-up was identical to the one described by Pont et al. (2007).

Table 2 includes the column with spectroscopic status resulting from the collected spectra. Only one candidate turned out to be a very promising planetary candidate revealing lowamplitude radial-velocity variation in phase with photometric ephemeris, namely OGLE-TR-211. It was then extensively observed during all following spectroscopic runs, allowing confirmation of its planetary nature. Three more candidates do not show significant radial velocity variations. Table 3 lists these objects and provides the upper limits on radial velocity semiamplitudes, $K$, at the $2-\sigma$ level, the corresponding maximum 
Table 2. Planetary and low-mass object transit candidates from the OGLE Campaign \#5 conducted in 2005.

\begin{tabular}{ccccccll}
\hline \hline Name & RA & $\begin{array}{c}\text { Dec } \\
{[2000]}\end{array}$ & $\begin{array}{c}\text { Period } \\
{[\text { days }]}\end{array}$ & $\begin{array}{c}T_{\mathrm{c}} \\
{[\mathrm{Hel} . J \mathrm{JD}]}\end{array}$ & $\begin{array}{c}I \\
{[\mathrm{mag}]}\end{array}$ & $\begin{array}{c}\text { Depth } \\
{[\mathrm{mag}]}\end{array}$ & Status \\
\hline OGLE-TR-201 & $10: 48: 31.61$ & $-62: 01: 01.8$ & 2.3680 & 2453404.860 & 15.6 & 0.016 & fast rotator \\
OGLE-TR-202 & $10: 46: 06.06$ & $-61: 52: 11.3$ & 1.6545 & 2453404.438 & 13.6 & 0.017 & not observed \\
OGLE-TR-203 & $10: 49: 32.04$ & $-61: 35: 38.2$ & 3.3456 & 2453406.178 & 15.6 & 0.014 & not observed \\
OGLE-TR-204 & $10: 47: 39.44$ & $-61: 19: 00.8$ & 3.1097 & 2453405.515 & 14.8 & 0.026 & SB2 \\
OGLE-TR-205 & $10: 45: 53.40$ & $-61: 09: 22.1$ & 1.7501 & 2453404.601 & 16.0 & 0.015 & not observed \\
OGLE-TR-206 & $10: 45: 22.00$ & $-61: 28: 28.8$ & 3.2658 & 2453403.246 & 13.8 & 0.006 & no variation \\
OGLE-TR-207 & $10: 40: 10.41$ & $-61: 53: 55.5$ & 4.8170 & 2453406.978 & 14.3 & 0.021 & SB2 \\
OGLE-TR-208 & $10: 39: 54.18$ & $-61: 58: 07.7$ & 4.5025 & 2453406.567 & 15.3 & 0.022 & SB2 \\
OGLE-TR-209 & $10: 40: 56.27$ & $-62: 14: 20.2$ & 2.2056 & 2453403.657 & 15.0 & 0.022 & no variation \\
OGLE-TR-210 & $10: 40: 44.21$ & $-62: 13: 21.6$ & 2.2427 & 2453403.012 & 15.2 & 0.032 & fast rotator \\
OGLE-TR-211 & $10: 40: 14.51$ & $-62: 27: 19.8$ & 3.6772 & 2453406.271 & 14.3 & 0.008 & planet \\
OGLE-TR-212 & $10: 43: 37.95$ & $-61: 45: 01.5$ & 2.2234 & 2453404.170 & 16.3 & 0.016 & blend? \\
OGLE-TR-213 & $10: 43: 00.85$ & $-61: 51: 10.2$ & 6.5746 & 2453403.301 & 15.3 & 0.036 & SB2 \\
OGLE-TR-214 & $10: 44: 27.96$ & $-61: 35: 35.7$ & 3.6010 & 2453403.090 & 16.5 & 0.023 & SB1 \\
OGLE-TR-215 & $10: 43: 48.69$ & $-61: 40: 00.3$ & 4.9237 & 2453407.616 & 14.8 & 0.016 & no variation \\
OGLE-TR-216 & $10: 42: 02.43$ & $-61: 20: 16.0$ & 1.9763 & 2453403.064 & 14.6 & 0.011 & blend? \\
OGLE-TR-217 & $10: 40: 59.73$ & $-61: 30: 38.4$ & 5.7208 & 2453404.104 & 16.1 & 0.037 & no ccf \\
OGLE-TR-218 & $10: 41: 12.74$ & $-61: 28: 20.8$ & 2.2488 & 2453404.697 & 14.5 & 0.020 & fast rotator \\
OGLE-TR-219 & $10: 40: 53.40$ & $-61: 43: 15.1$ & 9.7466 & 2453405.683 & 15.1 & 0.032 & SB2 \\
\hline
\end{tabular}

Notes: SB1 - single line spectroscopic binary; SB2 - double line spectroscopic binary; no cef - lack of the cross-correlation function.

Table 3. OGLE transit candidates with no significant radial velocity variation.

\begin{tabular}{cccc}
\hline \hline Object & $\begin{array}{c}K \text { limit } \\
{[\mathrm{m} / \mathrm{s}]}\end{array}$ & $\begin{array}{c}\text { Mass limit } \\
{\left[M_{\text {Jup }}\right]}\end{array}$ & $\begin{array}{c}\text { Radius limit } \\
{\left[R_{\text {Jup }}\right]}\end{array}$ \\
\hline OGLE-TR-206 & $<59$ & $<0.42$ & $>0.6$ \\
OGLE-TR-209 & $<16$ & $<0.16$ & $>0.8$ \\
OGLE-TR-215 & $<63$ & $<0.52$ & $>1.5$ \\
\hline
\end{tabular}

mass of a potential planet for a $1 M_{\odot}$ host star, and the lower limit on the planetary radius (i.e. central transit) for such a primary.

\section{OGLE-TR-211 candidate}

OGLE-TR-211 turned out to be the most promising candidate for a transiting planetary system from the OGLE Campaign \#5 sample. After the initial spectroscopic follow-up observations confirming this status, it was decided to allocate a considerable amount of observing time to a precise characterization of this object. OGLE-TR-211 is relatively bright $(I \approx 14.3 \mathrm{mag})$ in the OGLE sample. Its equatorial coordinates are listed in Table 2, while Fig. 1 shows the finding chart $-2^{\prime} \times 2^{\prime}$ OGLE I-band image. OGLE-TR-211 is listed in the 2MASS and DENIS catalogs as 2MASS10401438-6227201 and J104014.3-622720, respectively.

\subsection{Photometry}

The original OGLE 2005 season photometric data covered eight partial transits. When folded with the period of 3.677 days, they revealed a clear transit about 8 mmag deep. The estimated lower limit of the transiting companion radius was $0.12 R_{\odot}$ making OGLE-TR-211 a very good candidate for a transiting planet.

Due to the limited ( $\approx 3$ months long) duration of the OGLE campaign, the photometric period has a limited accuracy of $\approx 10^{-4} \mathrm{P}$ or worse in the case of low depth transits. In order to

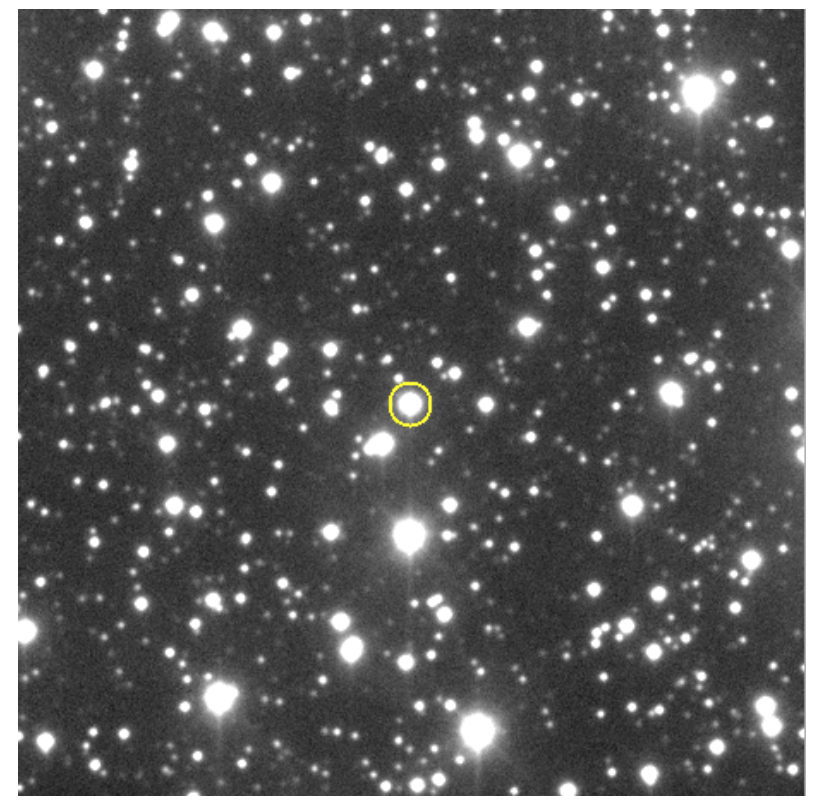

Fig. 1. Finding chart for OGLE-TR-211. Size of the $I$-band OGLE subframe is $120^{\prime \prime} \times 120^{\prime \prime}$, North is up and East to the left.

confirm the presence of transits in OGLE-TR-211 and refine the ephemeris, OGLE observed the star in the next observing seasons: 2006 and 2007. The first additional transit data points were collected in January 2006, providing a necessary confirmation and update of the photometric orbit for the coming spectroscopic follow-up observations. Up to June 2007 eight additional transits of OGLE-TR-211 were covered. Thanks to the over two yearlong baseline, these observations allowed us to derive precise photometric ephemeris of OGLE-TR-211, as listed in Table 5 (see Sect. 4.4).

The very small depth of transits combined with $\approx 5 \mathrm{mmag}$ accuracy of individual OGLE measurements makes determining the precise shape of transit difficult. Therefore, similarly to other cases of OGLE transiting planets, it was decided to conduct a 


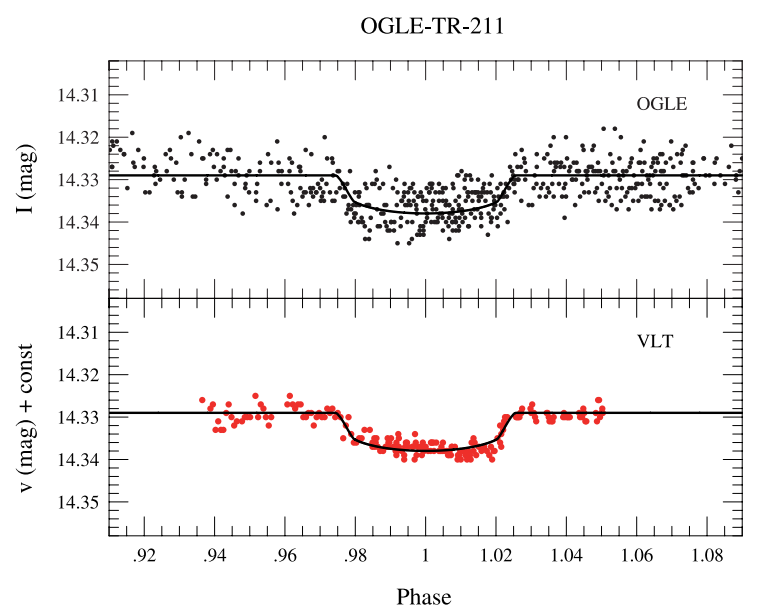

Fig. 2. Upper panel: OGLE light curve of OGLE-TR-211, lower panel: VLT observations. The continuous line represents the best model of the transit.

photometric follow-up of this object on a much larger telescope. Three photometric runs on VLT with the FORS camera were performed in the period of March/June 2006.

The first-run VLT images of OGLE-TR-211 were obtained on March 16, 2006 through a $V$-band filter with the exposure time of $8 \mathrm{~s}$. They cover the pre-ingress, ingress, and bottom of the transit. Unfortunately the egress of the transit was missed. Two additional runs were carried out on May 25, 2006 and June 30, 2006. In these cases observations were obtained through $V$ and $R$ filters and the exposure time varied from 25 to $80 \mathrm{~s}$ depending on seeing. Unfortunately, both of these runs cover a practically non-variable part of the transit light curve. The May observations show only the flat bottom of the transit with only a small trace of starting egress at the very end. The June observations cover only the post transit light curve with the very end of egress at the beginning. Thus, the full reconstruction of the precise VLT light curve of OGLE-TR-211 became difficult.

The VLT data were reduced using the OGLE data pipeline (Udalski 2003), based on the difference image analysis (DIA) method. Because the March 2006 observations that defined a considerable part of the transit shape were obtained in the $V$-band, only $V$-band images from the remaining observing runs were used. To have similar time resolution and to lower the scatter of the March observations, these data were binned to have an effective resolution of $3 \mathrm{~min}$. The March light curve indicates that the transit depth is about $8 \mathrm{mmag}$ - practically identical with what resulted from OGLE observations, which is reassuring. Therefore the May data were phased with the photometric period resulting from OGLE observations and adjusted in magnitude scale in such a way that the observed bottom of the transit corresponds to the level of the bottom observed in March 2006. Similarly, the June photometry was shifted so the post-egress part of transit corresponds to the pre-ingress part observed in March. The lower panel of Fig. 2 shows the reconstructed VLT light curve of OGLE-TR-211, while the OGLE light curve is presented in the upper panel.

The VLT data were also reduced using the DecPhot PSF fitting software (Gillon et al. 2006). While the results were generally similar, this photometry was not used for further analysis because of difficulties with the normalization between runs.
Table 4. Radial velocity measurements for OGLE-TR-211.

\begin{tabular}{ccccccc}
\hline \hline $\begin{array}{c}\text { Date } \\
{[\mathrm{JD}-2450000]}\end{array}$ & Phase $\left.\begin{array}{c}V R \\
{\left[\mathrm{~km} \mathrm{~s}^{-1}\right]}\end{array}\right] R_{\text {rectified }}\left[\begin{array}{c}\sigma_{\mathrm{VR}} \\
{\left[\mathrm{km} \mathrm{s}^{-1}\right]}\end{array}\right.$ & $\begin{array}{c}B S \\
{\left[\mathrm{~km} \mathrm{~s}^{-1}\right]}\end{array}$ & $\begin{array}{c}\sigma_{\mathrm{BS}} \\
{\left[\mathrm{km} \mathrm{s}^{-1}\right]}\end{array}$ \\
\hline 3793.81192 & 0.3889 & 18.784 & 18.784 & 0.055 & 0.132 & 0.071 \\
3794.75446 & 0.6452 & 18.866 & 18.866 & 0.053 & 0.073 & 0.069 \\
3852.50001 & 0.3487 & 18.720 & 18.720 & 0.053 & 0.007 & 0.033 \\
3853.51724 & 0.6254 & 18.875 & 18.875 & 0.053 & -0.055 & 0.016 \\
3854.54151 & 0.9039 & 18.841 & 18.841 & 0.051 & 0.038 & 0.028 \\
3855.50251 & 0.1652 & 18.711 & 18.711 & 0.055 & -0.020 & 0.088 \\
3855.59958 & 0.1916 & 18.816 & 18.816 & 0.060 & 0.025 & 0.034 \\
3858.49787 & 0.9798 & 18.906 & 18.906 & 0.054 & 0.027 & 0.058 \\
3881.57542 & 0.2556 & 18.766 & 18.766 & 0.053 & - & - \\
4143.83440 & 0.5753 & 18.860 & 18.860 & 0.046 & -0.004 & 0.018 \\
4144.71247 & 0.8141 & 18.911 & 18.911 & 0.046 & -0.043 & 0.032 \\
4145.72709 & 0.0900 & 18.762 & 18.762 & 0.048 & 0.249 & 0.100 \\
4149.81577 & 0.2019 & 18.681 & 18.681 & 0.047 & -0.146 & 0.031 \\
4150.77874 & 0.4638 & 18.891 & 18.891 & 0.046 & -0.070 & 0.122 \\
4203.55370 & 0.8156 & 18.745 & 18.925 & 0.046 & 0.038 & 0.052 \\
4204.51070 & 0.0759 & 18.623 & 18.803 & 0.046 & -0.036 & 0.058 \\
4205.59031 & 0.3695 & 18.657 & 18.837 & 0.046 & -0.043 & 0.047 \\
4207.70041 & 0.9433 & 18.658 & 18.838 & 0.046 & 0.073 & 0.061 \\
4208.68020 & 0.2097 & 18.578 & 18.758 & 0.046 & 0.074 & 0.051 \\
4209.61470 & 0.4639 & 18.517 & 18.697 & 0.049 & -0.118 & 0.074 \\
\hline
\end{tabular}

\subsection{Radial velocity follow-up}

Twenty high-resolution spectroscopic observations of OGLETR-211 were obtained with the FLAMES/UVES spectrograph on VLT during four spectroscopic follow-up runs. The strategy of observations and reduction procedures were identical to the description in Pont et al. (2007). The resulting radial velocities of OGLE-TR-211 with their errors are listed in Table 4. The errors include additional $45 \mathrm{~m} / \mathrm{s}$ term derived from the estimation of stability of the instrument and added in quadrature to the photon noise radial velocity errors to account for possible systematic errors.

After two 2006 runs, it was clearly found that the radial velocities of OGLE-TR-211 follow the photometric period and reveal $\approx 100 \mathrm{~m} / \mathrm{s}$ semi-amplitude variation in appropriate phase with planetary interpretation. However, during the last 2007 April run, while this variation was clearly seen, the $\gamma$ zero point of radial velocities was shifted by about $180 \mathrm{~m} / \mathrm{s}$, which is more than $3-\sigma$ of the typical accuracy of observations. This is a considerably larger shift than the shifts noted by Pont et al. (2007) during the analysis of the recently discovered transiting planet OGLE-TR-182b.

Although it cannot be fully ruled out that the shift has some instrumental origin, it is more likely that it corresponds to a real change in the average radial velocity. Unfortunately, the collected dataset is too limited to draw any sound conclusions about its origin. We can only speculate that it may be caused by the presence of an additional companion in the OGLE-TR-211 system having a wide orbit with a period of several years. Additional spectroscopic observations are necessary to verify this interpretation.

For the purpose of characterizing the planetary companion of OGLE-TR-211, the $\gamma$ velocity was fitted separately for each observing run for a preliminary fit of the spectroscopic orbit. Only the shift of $\gamma$ velocity of the 2007 April run turned out to be statistically significant. It was subtracted from original radial velocities for the final fit presented in the upper panel of Fig. 3. Rectified radial velocities of OGLE-TR-211 are also listed in Table 4. 


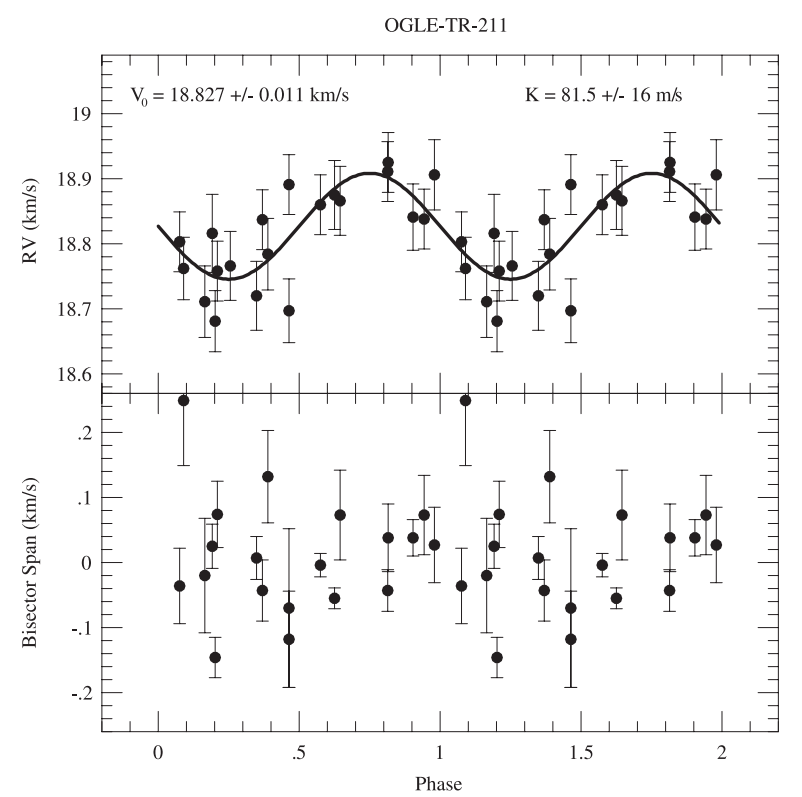

Fig. 3. Upper panel: radial velocity observations for OGLE-TR-211, phased with the photometric transit ephemeris. Solid line represents the best fit assuming no eccentricity. Two periods are presented for clarity. Lower panel: bisector span values plotted as a function of phase for testing blending scenarios.

Because blending of an eclipsing star with a bright third light source can cause radial velocity variations comparable to a planetary signal, line profiles of the spectra of OGLE-TR-211 were examined for the correlation of the average line bisector spans (BS - see the last columns of Table 4) with phase and observed radial velocities (e.g., Santos et al. 2002; Torres et al. 2004). No such correlation was found, allowing us to rule out blending scenario (lower panel of Fig. 3).

\subsection{Host star spectroscopy}

To derive parameters of the host star of OGLE-TR-211, highresolution spectra were obtained with the UVES spectrograph on the VLT working in the slit mode on May 7 and 8, 2007. The spectra were summed so the coadded spectrum reached an $\mathrm{S} / \mathrm{N}$ of about 100 in the $\lambda \approx 6707 \AA$ lithium line region. Measurements followed the method described in Santos et al. (2006), and the results are listed in Table 5. Temperature and gravity indicates that the host star is a dwarf of F7-8 spectral type and metallicity somewhat larger than solar.

\subsection{OGLE-TR-211 parameters}

The parameters of OGLE-TR-211 were derived with the standard method used in the case of the previous OGLE planets (Bouchy et al. 2004; Pont et al. 2007). The OGLE and VLT photometry with a red noise value equal to the photon noise was fitted for $P, T, b, R / R_{*}$, and $a / R_{*}$. The eccentricity of the orbit in the final fitting was assumed to be zero, as practically all the observed orbits of short period transiting planets are circularized by tidal interactions. Also our preliminary fit of the radial velocity curve with the orbital period fixed at the photometric value yielded eccentricity $e=0.16 \pm 0.22$, consistent with circular orbit at better than 1- $\sigma$ level. Model parameters with their $1-\sigma$ errors from chi-square analysis including the effect of correlated noise are listed in Table 5.
Table 5. Parameters of the OGLE-TR-211 system.

\begin{tabular}{|c|c|}
\hline Period [days] & $3.67724 \pm 0.00003$ \\
\hline Transit epoch [Hel. JD] & $2453428.334 \pm 0.003$ \\
\hline Transit duration [days] & $0.1838 \pm 0.0055$ \\
\hline$V R$ semi-amplitude $[\mathrm{m} / \mathrm{s}]$ & $82 \pm 16$ \\
\hline Semi-major axis [AU] & $0.051 \pm 0.001$ \\
\hline Orbit-radius ratio $\left[a / R_{*}\right]$ & $6.75^{+0.23}$ \\
\hline Radius ratio $\left[R / R_{*}\right]$ & $0.085 \pm 0.004$ \\
\hline$b$ & $0.68_{-0.08}^{+0.07}$ \\
\hline Orbital angle $\left[{ }^{\circ}\right]$ & $>82.7(1-\sigma)$ \\
\hline Light Curve Model: & \\
\hline OGLE rms [mmag] & 4.1 \\
\hline VLT rms [mmag] & 1.6 \\
\hline $\begin{array}{l}\text { Radial Velocity Model: } \\
\text { rms }[\mathrm{m} / \mathrm{s}]\end{array}$ & 48 \\
\hline$T_{\text {eff }}[\mathrm{K}]$ & $6325 \pm 91$ \\
\hline $\log g$ & $4.22 \pm 0.17$ \\
\hline$\eta\left[\mathrm{km} \mathrm{s}^{-1}\right]$ & $1.63 \pm 0.21$ \\
\hline$[\mathrm{Fe} / \mathrm{H}]$ & $0.11 \pm 0.10$ \\
\hline Stellar radius $\left[R_{\odot}\right]$ & $1.64_{-0.07}^{+0.21}$ \\
\hline Stellar mass $\left[M_{\odot}\right]$ & $1.33 \pm 0.05$ \\
\hline Planet radius $\left[R_{\mathrm{Jup}}\right]$ & $1.36_{-0.09}^{+0.18}$ \\
\hline Planet mass $\left[M_{\mathrm{Jup}}\right]$ & $1.03 \pm 0.20$ \\
\hline
\end{tabular}

Then the radius and mass of the host star were estimated with the maximum-likelihood method combining the observed spectroscopic parameters of the host star, transit parameters obtained from the light curve fit and Girardi et al. (2002) stellar evolution models. Table 5 lists the parameters of the host star and transiting planet in the OGLE-TR-211 system.

The transit shape is not precise enough to determine the inclination angle very precisely, mainly because of the effect of red noise with only partial transits measured with the VLT. Nevertheless, the size of the planet is relatively well-constrained thanks to the accuracy of the stellar spectroscopic parameters and the weak dependence of the planetary radius on the impact parameter for central transits.

The $\mathrm{O}-\mathrm{C}$ weighted rms scatter around the final photometric model of the OGLE-TR-211 system is equal to $\sigma=1.6 \mathrm{mmag}$ and $\sigma=4.1 \mathrm{mmag}$ for the VLT and OGLE dataset, respectively. The weighted rms of radial velocity observations relative to the best fit is $\sigma_{\mathrm{RV}}=48 \mathrm{~m} / \mathrm{s}$.

\section{Discussion}

Parameters of the transiting companion of the OGLE-TR-211 host star indicate that it is a planetary object belonging to the hot Jupiter class of extrasolar planets. It brings the total number of extrasolar planets discovered by the OGLE survey to seven - significantly lowering the discrepancy between the number of typical hot Jupiters and shortest period, very hot Jupiters in the OGLE sample of extrasolar transiting planets (Pont et al. 2005a; Gould et al. 2006).

OGLE-TR-211b orbits an F7-8 main sequence star. The system parameters closely resemble those of the recently discovered system HAT-P-6 (Noyes et al. 2007) with the only difference that the host star in OGLE-TR-211 is more metal abundant by $\approx 0.2$ dex. The position of the planet on the mass-orbital period diagram is similar to the position of other transiting exoplanets 


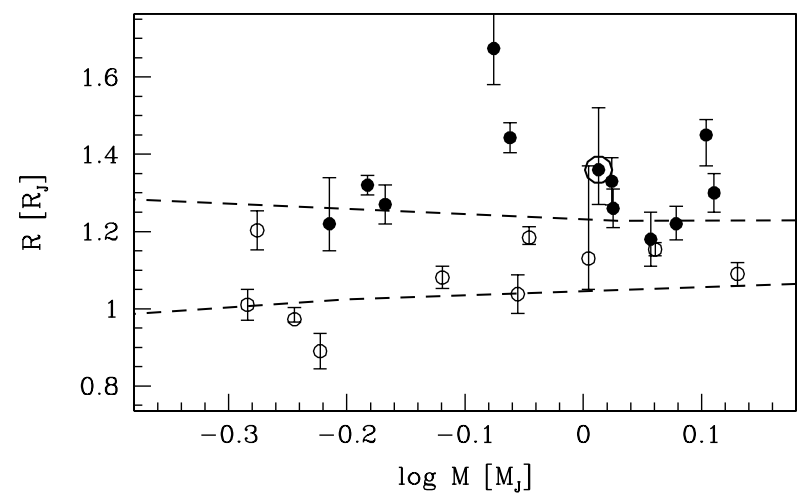

Fig. 4. Mass-radius diagram for transiting hot Jupiters $\left(0.5-1.5 M_{\mathrm{J}}\right)$. Closed symbols indicate host stars with $T_{\text {eq }}>1500 \mathrm{~K}$. OGLE-TR-211 is indicated by the circled dot. The lines show the models of Guillot (2005) in two extreme cases: cold gas planet with a $15 M_{\oplus}$ core and $T_{\text {eq }}=2000 \mathrm{~K}$ planet without a core. The six large planets with radius error bars above the upper models are all strongly irradiated.

with similar mass and period, supporting the relation noted first by Mazeh et al. (2005). Also, the surface gravity of OGLETR- $211 \mathrm{~b}$ of about $10.3 \mathrm{~m} / \mathrm{s}^{2}$ is consistent with the correlation between period and surface gravity noted by Southworth et al. (2007).

Recently Hansen \& Barman (2007) have proposed the divison of hot Jupiters into two classes based on their location on the (zero albedo) equilibrium temperature - Safronov number, $\Theta$, diagram. The position of OGLE-TR-211b in this diagram ( $T_{\text {eq }}=1720 \mathrm{~K}, \Theta=0.059$ ) implies that this planet belongs to the class I of hot Jupiters according to their classification.

While the mass of a new hot Jupiter OGLE-TR-211b is very similar to the mass of another hot Jupiter, OGLE-TR-182b, recently found by Pont et al. (2007), its radius is about $20 \%$ larger. The mean density of OGLE-TR-211b is equal to $0.54_{-0.24}^{+0.15} \mathrm{~g} / \mathrm{cm}^{3}$, placing it among a group of seven objects with the lowest densities: Tres-4, HD 209458, HAT-P-1, WASP-1, HAT-P-4, and HAT-P-6 (Kovács et al. 2007). All these objects have inflated radii compared to other transiting hot Jupiters of similar mass. Such planets pose a problem for the planetary models and are crucial objects for testing the proposed scenarios explaining "bloated" hot Jupiters (Burrows et al. 2007; Guillot et al. 2006). It is worth noting that the metallicity of the OGLE-TR-211 host star falls in the middle of the range of metallicities of "bloated" Jupiters host stars (recently increased significantly by the HATP-6 system) suggesting that metallicity cannot be the only parameter responsible for inflating the radii of hot Jupiters.

On the other hand, the characteristics of the OGLE-TR-211 system reinforce the association of "bloated" close-in giant planets with strong irradiation from the host star. Figure 4 shows the mass-radius diagram for the presently known (January 2008) transiting planets, classified according to their equilibrium temperature $^{1}$.

All anomalously large planets have equilibrium temperatures hotter than $1500 \mathrm{~K}$. This would require an increasingly unlikely coincidence if the anomalously large radii were explained by dynamical effects such as obliquity tide or eccentricity pumping by an unseen companion (Winn \& Holman 2005). It instead tends to favor explanations directly linking the excess of radius with the incident flux, such as proposed by Guillot et al. (2006) (that a fraction of the incident flux energy is converted to mechanical energy by a yet undetermined physical process) - over explanations with a more indirect causal relation, such as put forward by Burrows et al. (2007) and Chabrier \& Baraffe (2007) invoking increased opacity or internal density gradients.

Further additional high-accuracy photometric follow-up observations of the OGLE-TR-211 transits are necessary for better constraining the inclination and radius of the planet. Additional high-resolution spectroscopy planned in the 2008 observing season should lower any uncertainty about the planet mass. Although OGLE-TR-211, similar to other OGLE transiting planets, is too faint for the currently available facilities to successfully carry out some follow-up observations like IR photometry, the precise long-term transit timing is feasible allowing search for another planetary objects in the OGLE-TR-211 system.

Acknowledgements. We thank the anonymous referee for constructive remarks and comments that improved the paper. The OGLE project is partially supported by the Polish MNiSW grant N20303032/4275. W.G., D.M., G.P., M.T.R., and M.Z. gratefully acknowledge support for this work from the Chilean FONDAP Center of Astrophysics 15010003. N.C.S. acknowledges the support from Fundação para a Ciência e a Tecnologia, Portugal, in the form of a grant (reference POCI/CTE-AST/56453/2004), and support by the EC's FP6 and by FCT (with POCI2010 and FEDER funds), within the HELAS international collaboration.

\section{References}

Bouchy, F., Pont, F., Santos, N. C., et al. 2004, A\&A, 421, L13 Bouchy, F., Pont., F., Melo, C., et al. 2005, A\&A, 431, 1105 Burrows, A., Hubeny, I., Budaj, J., \& Hubbard, W. B. 2007, ApJ, 661, 502 Chabrier, G., \& Baraffe, I. 2007, ApJ, 661, L81

Charbonneau, D., Brown, T. M., Latham, D. W., \& Mayor, M. 2000, ApJ, 529, L45

Gillon, M., Pont, F., Moutou, C., et al. 2006, A\&A, 459, 249

Girardi, L., Bertelli, G., Bressan, A., et al. 2002, A\&A, 391, 195 Gould, A., Dorsher, S., Gaudi, B. S., \& Udalski, A. 2006, Acta Astron., 56, 1 Guillot, T. 2005, Ann. Rev. of Earth and Planetary Sciences, 33, 493 Guillot, T., Santos, N. C., Pont, F., et al. 2006, A\&A, 453, L21 Hansen, B. M. S., \& Barman, T. 2007, ApJ, 671, 861

Henry, G. W., Marcy, G. W., Butler, R. P., \& Vogt, S. S. 2000, ApJ, 529, L41 Konacki, M., Torres, G., Jha, S., \& Sasselov, D. D. 2003, Nature, 421, 507 Konacki, M., Torres, G., Sasselov, D. D., et al. 2004, ApJ, 609, L37 Konacki, M., Torres, G., Sasselov, D. D., \& Jha, S. 2005, ApJ, 624, 372 Kovács, G., Zucker, S., \& Mazeh, T. 2002, A\&A, 391, 369 Kovács, G., Bakos, G. Á., Torres, G., et al. 2007, ApJ, 670, L41 Kruszewski, A., \& Semeniuk, I. 2003, Acta Astron., 53, 241 Mazeh, T., Zucker, S., \& Pont, F. 2005, MNRAS, 356, 955

Noyes, R. W., Bakos, G. Á., Torres, G., et al. 2007, ApJ, 673, L79

Pont, F., Bouchy, F., Queloz, D., et al. 2004, A\&A, 426, L15 Pont, F., Bouchy, F., Melo, C., et al. 2005a, A\&A, 438, 1123 Pont, F., Melo, C., Bouchy, F., et al. 2005b, A\&A, 433, L21 Pont, F., Moutou, C., Bouchy, F., et al. 2006a, A\&A, 447, 1035 Pont, F., Zucker, S., \& Queloz, D. 2006b, MNRAS, 373, 231 Pont, F., Tamuz, O., Udalski, A., et al. 2007, A\&A, in press [arXiv: astro-ph/0710.5278]

Santos, N. C., Mayor, M., Naef, D., et al. 2002, A\&A, 392, 215

Santos, N. C., Pont, F., Melo, C., et al. 2006, A\&A, 450, 825 Southworth, J., Wheatley, P. J., \& Sams, G. 2007, MNRAS, 379, L11 Sozzetti, A., Torres, G., Charbonneau, D., et al. 2007, ApJ, 664, 1190 Torres, G., Konacki, M., Sasselov, D. D., \& Jha, S. 2004, ApJ, 614, 979 Udalski, A. 2003, Acta Astron., 53, 291

Udalski, A., Paczyński, B., Żebruń, K., et al. 2002a, Acta Astron., 52, 1 Udalski, A., Żebruń, K., Szymański, M., et al. 2002b, Acta Astron., 52, 115 Udalski, A., Szewczyk, O., Żebruń, K., et al. 2002c, Acta Astron., 52, 317 Udalski, A., Pietrzyński, G., Szymański, M., et al. 2003, Acta Astron., 53, 133 Udalski, A., Szymański, M. K., Kubiak, M., et al. 2004, Acta Astron., 54, 313 Winn, J. N., \& Holman, M. J. 2005, ApJ, 628, L159 